\title{
Preliminary study of the primary nozzle position of a supersonic air ejector with a constant-area mixing chamber
}

\author{
Jan $\mathrm{Kracik}^{1, *}$, and Vaclav Dvorak ${ }^{1}$ \\ ${ }^{1}$ Department of Power Engineering Equipment, Faculty of Mechanical Engineering, Technical University of Liberec, Studentska 2, \\ 46117, Liberec, the Czech Republic
}

\begin{abstract}
This work aims at investigating the primary nozzle position in a proposed supersonic air ejector device. The ejector is primarily made up of a supersonic primary nozzle, which is located in the axis of the ejector, a suction chamber or secondary stream inlet, a mixing chamber and a diffuser. The ejector design allows to translate the primary nozzle in the axis direction and fix it in a chosen distance from the beginning of the mixing chamber and hence influence the secondary mass flow rate. In a limit case, it is possible to set the nozzle to such a position where no secondary flow occurs. If we ignore the case where no secondary flow occurs, five different nozzle distances have been investigated in this paper. Some cases seem to be alike and there are no significant dissimilarities between them. Courses of relative back-pressure ratio are carried out against the entrainment ratio and transition between on-design and off-design regimes is determined. Measurements of the mixed flow based on the standard ISO 5167 are performed by means of orifice plate method. In addition, a comparison between experiments and simulations performed by Ansys Fluent software is presented in order to indicate further improvements to the numerical model.
\end{abstract}

\section{Introduction}

These days, when environment-friendly technologies are highly desired, devices operating with low energy consumption should get more attention. Ejectors are not an exception. These devices, with their efficiency generally lower than 30 per cent, require more research effort and a detailed investigation. The low efficiency is the greatest drawback of these devices. On the other hand, there are some advantages which make the use of these devices more attractive. Ejectors are commonly used for their simplicity, reliability and lack of moving parts. In this regard, special attention should be devoted to the improvement of their design. Ejectors can be used for both incompressible and compressible fluids. But it should be mentioned that the flow and especially the mixing of compressible fluids is very difficult to analyse. Ejectors are widely used in applications where sufficient amount of the working fluid or some waste thermal energy is available. They have established a long history in refrigeration. In this regard, they can be employed in connection with solar-driven systems or absorption systems. Refrigeration is probably one of the most important application areas of ejectors. Other possible applications of ejectors are thrust augmentation of aircraft propulsion systems, improving turbine performance, transport of solid particles and more. To spread out the use of ejectors in other commercial sectors, more research in the field of design methods is needed.

\footnotetext{
* Corresponding author: jan.kracik@tul.cz
}

Ejectors operating with incompressible fluids are often called as jet-pumps. Ejectors operating with compressible fluids are sometimes further distinguished as ejectors and injectors. The term ejector is used for a device that evacuates a vessel and an injector for a device that pumps mixed fluids against high pressure. One of the first works on ejectors was performed by Keenan [1].

Two major cases of mixing in ejector based on work [2] are distinguished: the constant-pressure mixing and the constant-area mixing. It should be noted that designing of constant-pressure ejectors is very complex and causes many problems for researchers. It is caused by the condition of constant static pressure which requires a precise determination of mixing chamber geometry to obtain constant static pressure. On the other hand, ejectors designed based on constant-pressure mixing seem to give superior performance in most cases. However, there has not been established a definite link between the performance of constant-area ejectors and constant-pressure ejectors yet. Another distinction of ejectors can be considered in terms of primary and secondary streams arrangement. The primary stream can either be supplied in the ejector axis and secondary stream is sucked on the periphery of the mixing chamber or primary stream enters the periphery of the mixing chamber and the secondary stream is sucked in the ejector axis. The latter design is commonly used for wind tunnel propulsions and can have two possible arrangements. The first arrangement uses a slot primary nozzle. The second one uses several primary nozzles and 
was investigated in works [3-6]. Many theoretical, experimental and numerical researches have been carried out to obtain the performance of ejector correctly so far.

In Fig. 1, the performance line of supersonic ejectors divided into three sections is shown. Generally, supersonic ejectors are designed for working within a range of back-pressures which corresponds to on-design section. This section is recognised as a part with a maximal entrainment ratio and it is also a sign that both primary and secondary flow are choked. There is no dependency of entrainment ratio on back-pressure in this section. The second section is often labelled as an offdesign section. In this case, there is a significant dependency of entrainment ration on back-pressure. The last section is a section of reverse flow, i.e. malfunction working regime, and we always try to avoid working ejectors in this regime.

The ejector under investigation in this paper was proposed in work [7]. The geometry of the proposed ejector is shown in Fig. 2. The primary nozzle is designed to generate exit Mach number of 2.06. The ejector is aimed to be utilized for future investigations of optimal shape of the wall in the mixing chamber region, thus finding an optimum nozzle position $(\mathrm{x})$ is needed. At present, the mixing chamber has constant-area shape, which will also be considered as an initial shape for possible future optimization. Air has been used as a working fluid. Optimization is intended to be done with a 3D model. Before optimization, a comprehensive study and research on this ejector is desired.

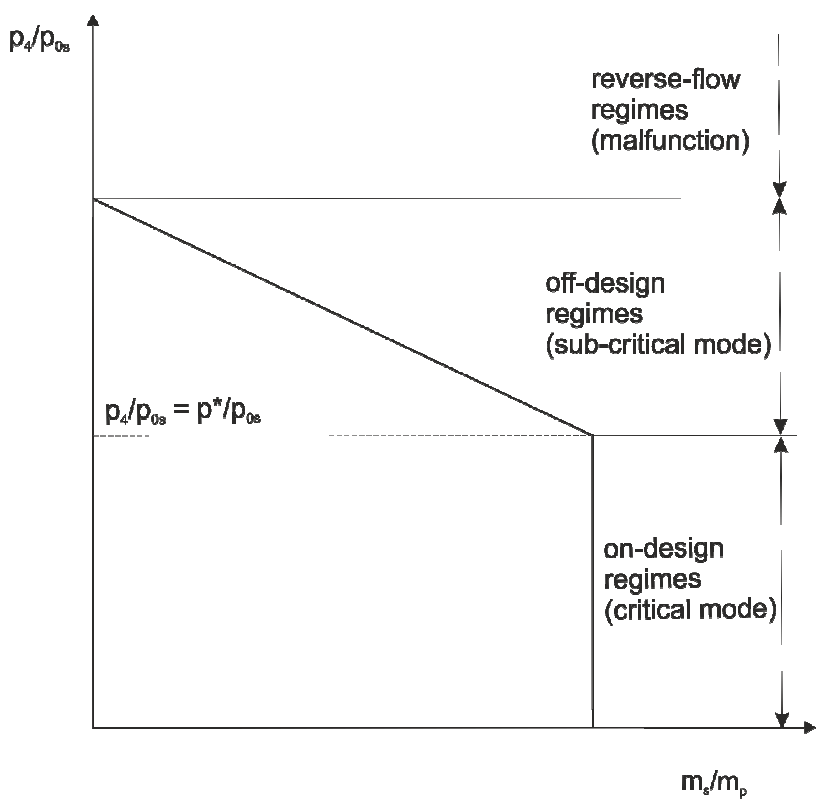

Fig. 1. Supersonic ejector operational regimes.

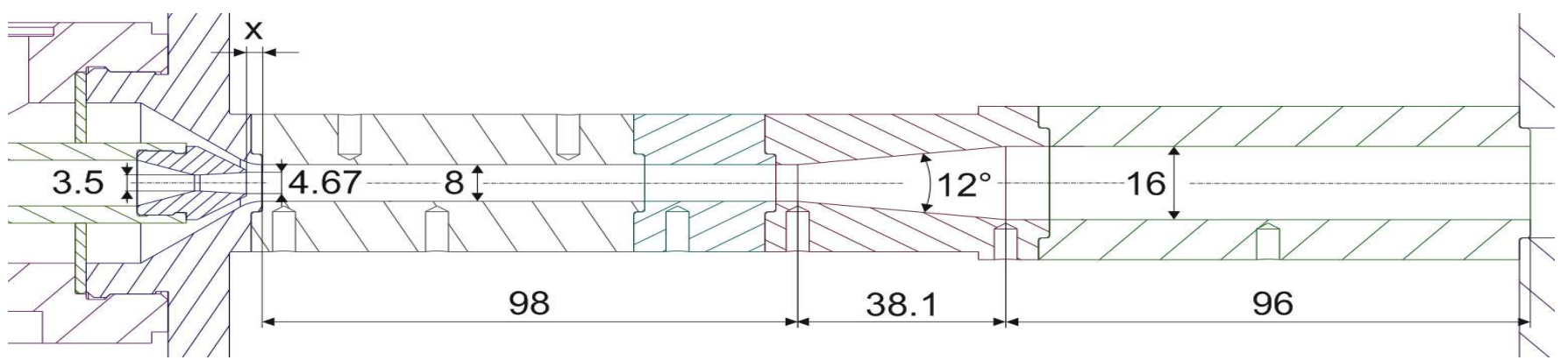

Fig. 2. Ejector geometry with main dimensions in millimetres. Dimension $\mathrm{x}$ denotes position of the primary nozzle.

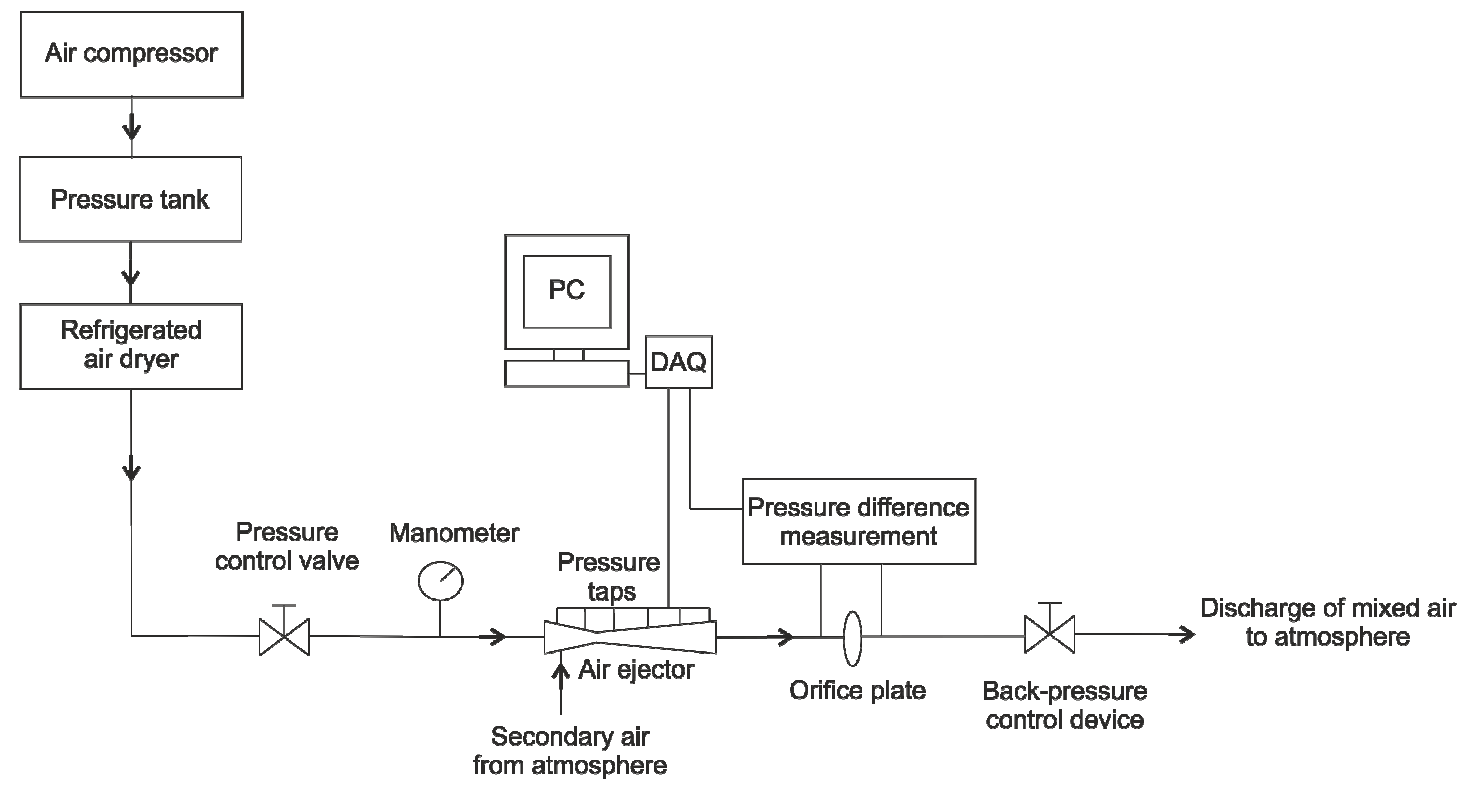

Fig. 3. Schematic diagram of experimental apparatus. 


\section{Experimental setup}

In order to get performance lines of the ejector, the entrainment ratio must be calculated. The entrainment ratio is defined as a ratio of mass flow rates, i.e.

$$
\omega=\frac{\dot{m}_{s}}{\dot{m}_{p}}
$$

where subscript $p$ denotes primary flow and $s$ stands for secondary flow, i.e. induced or sucked flow.

With regards to the experimental device arrangement shown in Fig. 3, the pressure difference of the mixed flow on the orifice plate is measured in case that the secondary flow is allow to be sucked into the mixing chamber. In the same manner and for no secondary flow, i.e. for closed secondary air inlet, the pressure difference of the primary flow can be measured. Secondary mass flow rate is then calculated as a difference of both and is expressed by

$$
\dot{m}_{s}=\dot{m}_{\text {total }}-\dot{m}_{p}
$$

The primary and total mass flow rate are calculated according to the ISO 5167 standard as follows

$$
\dot{m}=\frac{C}{\sqrt{1-\beta^{4}}} \varepsilon \frac{\pi}{4} d^{2} \sqrt{2 \Delta p \rho_{1}},
$$

where $\Delta p=p_{2}-p_{1}$ is pressure difference between the points located downstream and upstream the orifice plate respectively, $C$ is a discharge coefficient, $\varepsilon$ is an expansion coefficient, $d$ is the orifice diameter, $\rho_{1}$ is density upstream the orifice plate and $\beta$ is a relation between orifice and pipe diameter $D$ and is defined as

$$
\beta=\frac{d}{D}
$$

Since a discharge coefficient is usually not known, the iterative procedure is needed for solving equation (3). The relative error between the calculated primary mass flow rate based on the introduced method and the primary mass flow rate obtained from numerical analysis is about $3 \%$. Even smaller relative error, approximately $0.1 \%$, is between the mass flow rate calculated according to relation (3) and the primary mass flow rate determined by theory.

Piezoelectric pressure sensors are used for acquisition of total pressure of primary air $p_{0 p}$ and backpressure downstream the diffuser $p_{b}$. Since the secondary air is sucked directly from atmosphere, total pressure of the secondary air is considered to be equal to the barometric pressure in the laboratory. Fig. 4 presents the ejector used for experiments. The total pressure of primary air is $p_{0 p}=397 \mathrm{kPa}$ with temperature $T_{0 p}=300 \mathrm{~K}$. The total pressure of secondary air is equal to the ambient air pressure in laboratory, i.e. $p_{0 s}=p_{a}=97 \mathrm{kPa}$, temperatures are $T_{0 s}=T_{0 p}=300 \mathrm{~K}$. Relative back-pressure is changed within range $(0 \div 65)$ $\mathrm{kPa}$.

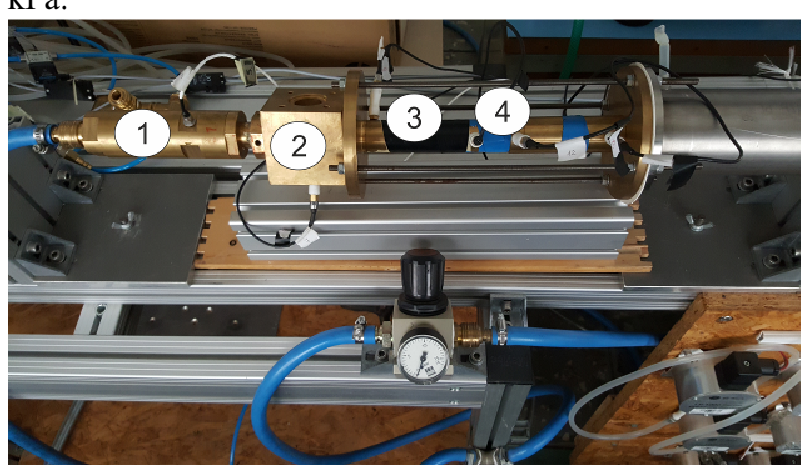

Fig. 4. Photo of the experimental ejector with its main parts: 1 - primary air inlet with the nozzle, 2 - secondary air inlet, 3 - mixing chamber, 4 - diffuser.

\section{Numerical approach}

The flow in the ejector is described by the compressible form of the conservation equations since they are more suitable for describing flows where density variations play a significant role. The influence of thermodynamics and transport properties of air was not proved to be significant, thus thermodynamics and transport properties are considered as constants during simulations. The air is modelled according to the perfect gas law. In order to limit complexity of the numerical model, two-dimensional axisymmetric models of the ejector is used to obtain numerical data, especially for calculating entrainment ratio. Although the steady state is assumed within the ejector, governing equations with an unsteady term are solved with a time marching technique. The governing equations can be written in their Cartesian form as follows:

$$
\begin{gathered}
\frac{\partial \rho}{\partial t}+\frac{\partial}{\partial x_{i}}\left(\rho u_{i}\right)=0 \\
\frac{\partial}{\partial t}\left(\rho u_{i}\right)+\frac{\partial}{\partial x_{j}}\left(\rho u_{i} u_{j}\right)=-\frac{\partial}{\partial x_{j}}\left(p \delta_{i j}\right)+\frac{\partial \tau_{i j}}{\partial x_{j}} \\
\frac{\partial}{\partial t}\left(\rho e_{0}\right)+\frac{\partial}{\partial x_{j}}\left(\rho u_{j} e_{0}+u_{j} p+q_{j}-u_{i} \tau_{i j}\right)=0 \\
p=\rho r T
\end{gathered}
$$

and for a Newtonian fluid the viscous stress term is given by

$$
\tau_{i j}=\mu_{e f f}\left(\frac{\partial u_{i}}{\partial x_{j}}+\frac{\partial u_{j}}{\partial x_{i}}\right)-\frac{2}{3} \mu_{e f f} \frac{\partial u_{k}}{\partial x_{k}} \delta_{i j} .
$$

The governing equations are solved using the commercial CFD package Fluent provided by Ansys, Inc. Two-dimensional hybrid mesh with nearly 71,200 elements was created for calculations. However, most of 
the elements are quadrilaterals. Due to relatively small values of mass flow rates, double precision is used to get more precise solution. For flow variables a second-order upwind scheme is utilized. To take turbulence effects into account the k- $\omega$ SST turbulence model is used. This model is suitable for calculation of flows in supersonic ejectors as it was many times proved in previous works [8, 9 and 10]. For more information, see Ansys Fluent documentation [11].

\section{Validation}

In figures 5-9, comparison of experiment and numerical simulation in form of performance lines for different primary nozzle positions can be seen. In Fig. 5, performance lines for $1 \mathrm{~mm}$ are carried out to compare. Here can be seen relatively small discrepancies in ondesign region, i.e. for the lowest values of back-pressure. In this case, the maximal entrainment ratio obtained from experiments is about 0.36 . The performance lines for $2 \mathrm{~mm}$ show higher values of maximal entrainment ratio (Fig. 6). The maximal value for experiment is 0.74 , which is approximately twice as high as in the case for 1 $\mathrm{mm}$. However, the range of back-pressure to maintain this value is smaller. Fig. 7 to 9 show almost the same results for experiments and numerical analyses. For all three cases the maximal entrainment ratio is approximately 0.78 during measurements. The maximal entrainment ratio obtained from numerical analysis is a little higher (0.83). In Fig. 10, the performance lines for all five measured cases are carried out to compare. The same comparison for numerical analysis is shown in Fig. 11.

The relative error for all the investigated cases is depicted in Fig. 12. It is obvious for all the cases that for higher values of back-pressure the relative error is higher than $20 \%$. On the contrary, in the on-design region for lower back-pressures, the relative error lies within range $\pm 10 \%$.

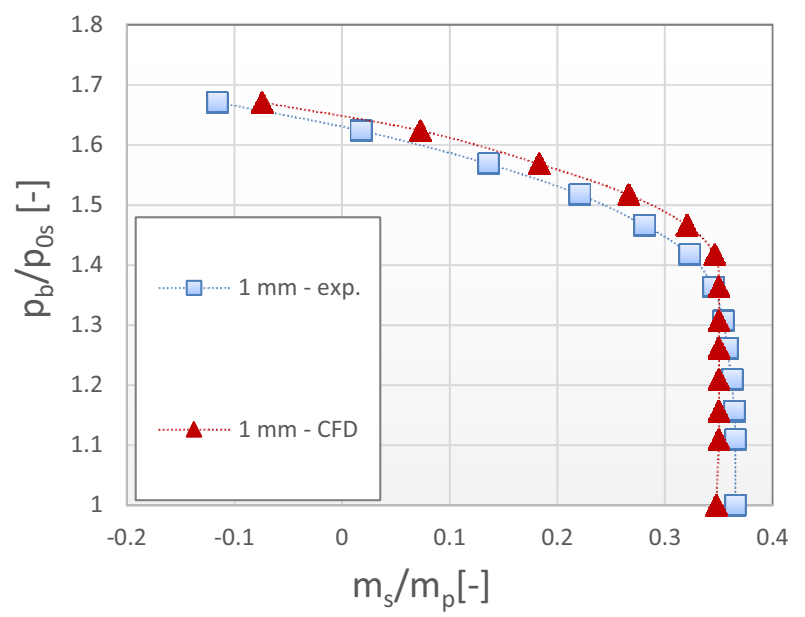

Fig. 5. Comparison between the experimental and numerical data for nozzle position $\mathrm{x}=1 \mathrm{~mm}$.

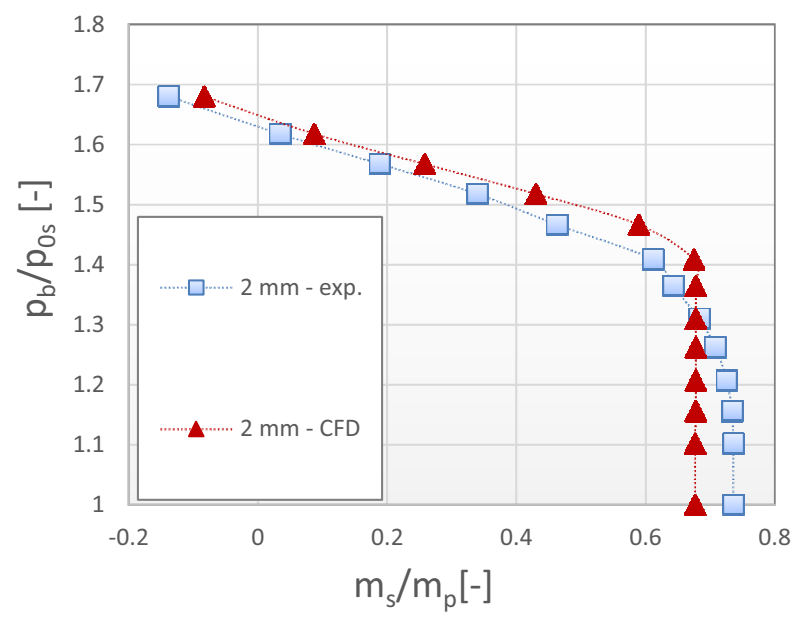

Fig. 6. Comparison between the experimental and numerical data for nozzle position $\mathrm{x}=2 \mathrm{~mm}$.

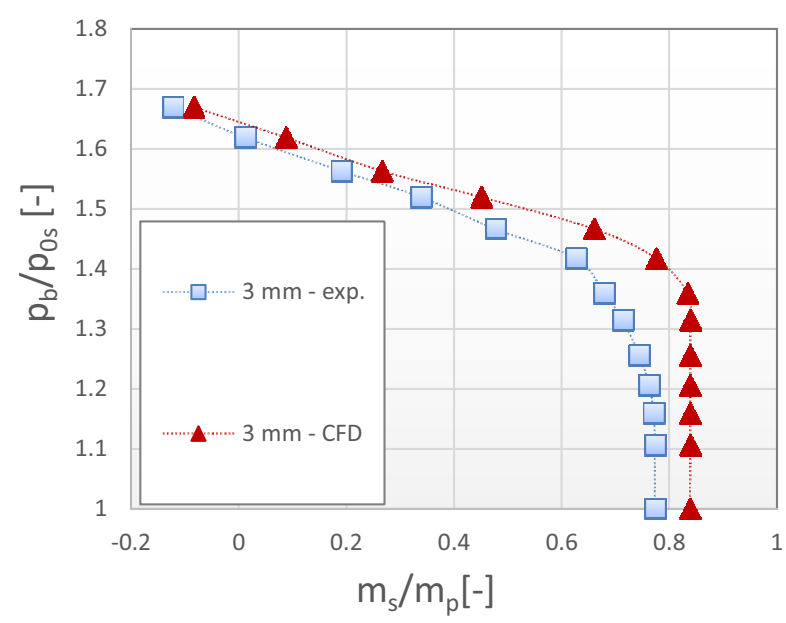

Fig. 7. Comparison between the experimental and numerical data for nozzle position $\mathrm{x}=3 \mathrm{~mm}$.

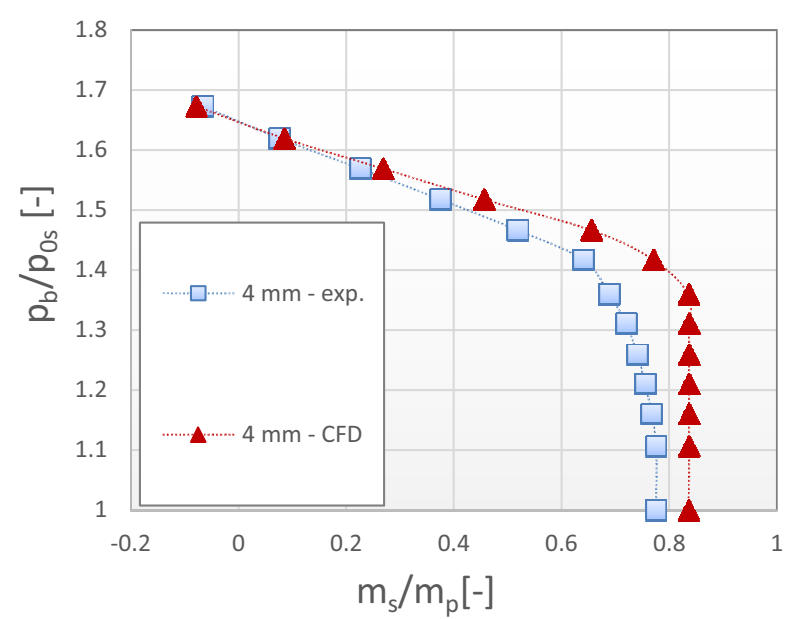

Fig. 8. Comparison between the experimental and numerical data for nozzle position $\mathrm{x}=4 \mathrm{~mm}$. 


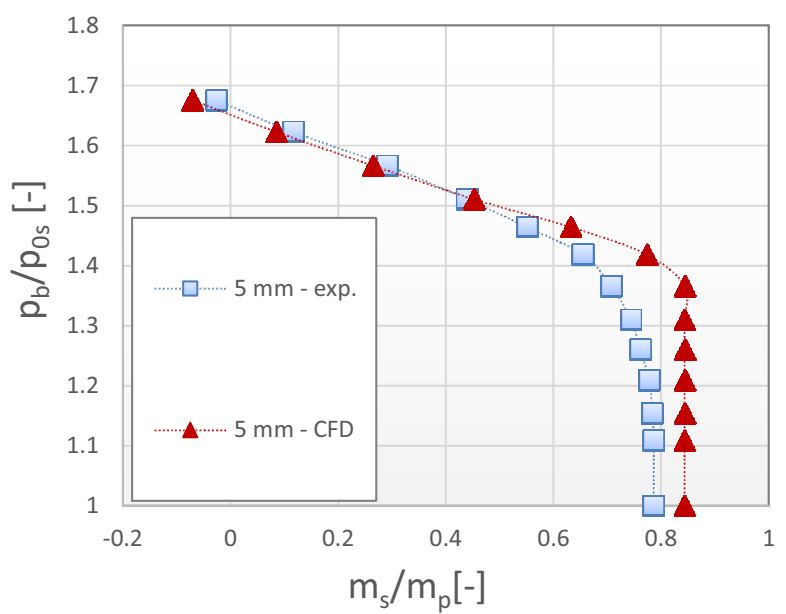

Fig. 9. Comparison between the experimental and numerical data for nozzle position $\mathrm{x}=5 \mathrm{~mm}$.

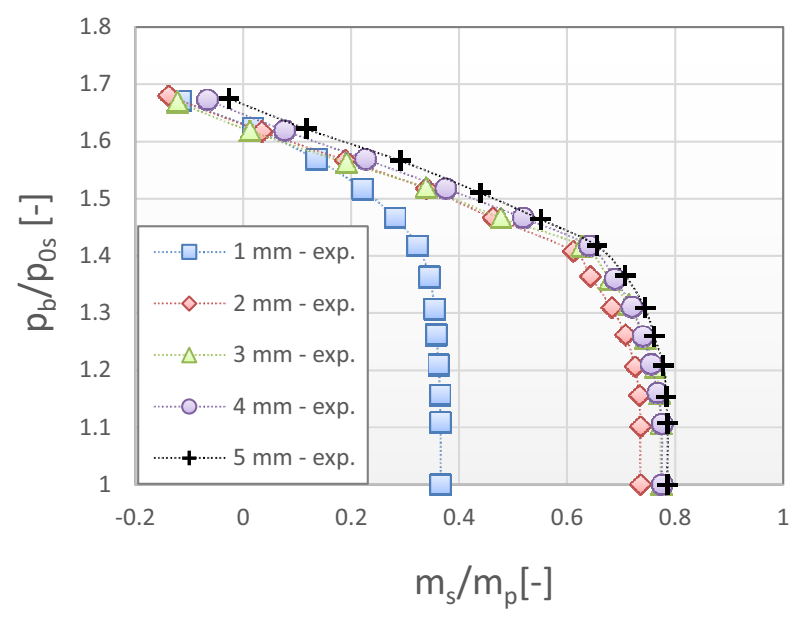

Fig. 10. Performance lines for all investigated cases obtained from experiment.

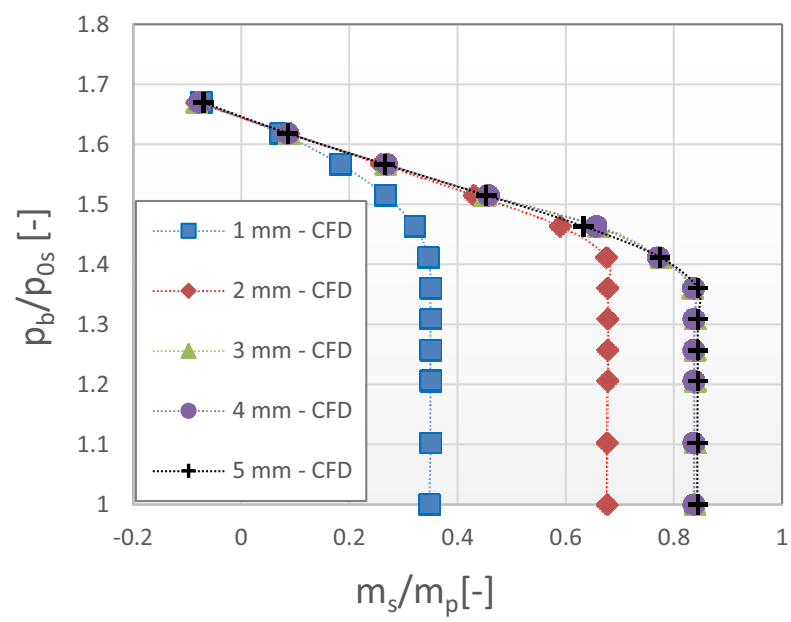

Fig. 11. Performance lines for all investigated cases obtained from numerical analysis.

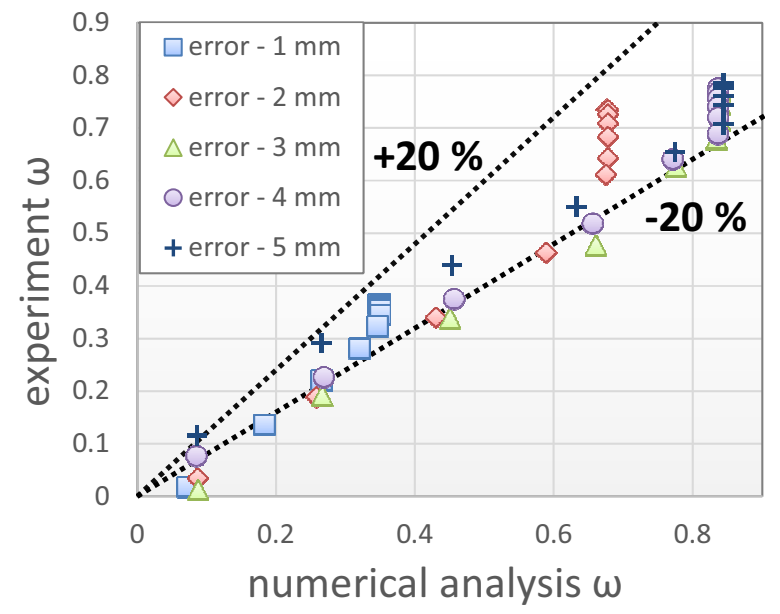

Fig. 12. Relative error between entrainment ratio $\omega$ obtained by the experiment and the numerical computation (CFD).

\section{Summary and conclusion}

The first experimentally obtained performance lines of a supersonic ejector with various primary nozzle positions have been presented in this paper. It turns out that from a certain distance of the primary nozzle from the beginning of the mixing chamber there is no significant increase in the maximal entrainment ratio (on-design regime) and the cases for distance of 3,4 and 5 millimetres seem to be nearly the same. The measured maximal entrainment ratio is 0.78 for these cases.

The numerical results obtained on 2D axisymmetric models have been presented. The comparison with the experimental data shows some discrepancies, particularly in the off-design region. It could be caused by too much simplified numerical models or by other phenomena which occur for higher range of backpressure. On the other hand, the largest error between experimental and numerical results in the on-design region is less than $20 \%$ and most data points lies within $\pm 10 \%$ error. Anyway, there is a need for performing 3D calculations in the future. Furthermore, a comprehensive analysis of experimental and numerical data is necessary to understand flow processes that occur within the ejector, particularly the mixing chamber and diffuser sections.

This publication was written at the Technical University of Liberec as part of the project "Experimental and numerical investigation in applied fluid mechanics and energy devices, no. 21124" with the support of the Specific University Research Grant, as provided by the Ministry of Education, Youth and Sports of the Czech Republic in the year 2016.

\section{References}

1. J.H. Keenan, E.P. Neumann, A simple air Ejector, Journal of Applied Mechanics, Trans. ASME 64, pp A75 - A81 (1942)

2. J.H. Keenan, E.P. Neumann and F. Lustwerk, An Investigation of Ejector Design by Analysis and 
Experiment. Journal of Applied Mechanics, Trans. ASME 17 (1950), pp 299 - 309.

3. J. Kracik, V. Dvorak, Experimental and numerical investigation of an air to air supersonic ejector for propulsion of a small supersonic wind tunnel, EPJ Web of Conferences 92 (2015) 02038

4. J. Kracik, V. Dvorak, J. Kolar, Development of air to air ejector for supersonic wind tunnel, Web of Conferences 67 (2014) 02059

5. J. Kracik, Design of Supersonic Wind Tunnel Diploma thesis, In Czech, (2014)

6. V. Dvorak, Shape Optimization of Supersonic Ejector for Supersonic Wind Tunnel. Applied and Computational Mechanics, Plzen, (2010)

7. A. M. S. Guanlao, Design and verification of a test rig for research of supersonic ejectors, Diploma thesis, (2016)

8. Y. Bartosiewicz, Z. Aidoun, P. Desevaux, Y. Mercadier, Numerical and experimental investigations on supersonic ejectors, International Journal of Heat and Fluid Flow 26, pp $56-70$ (2005)

9. J. Kolar, V. Dvorak, Interaction of Shock Waves in Supersonic Ejector (Proceedings of the 27th Meeting of Thermodynamics and Fluid Mechanics Departments, Plzen, 2008)

10. J. Simak, Computation of the Flow and Interaction of Shock Waves in a 2D Supersonic Ejector (Colloquium Fluid Dynamics 2009, Prague)

11. Ansys Fluent 15.0 User's Guide, Ansys Inc. 